\title{
Focal dermal hypoplasia (Goltz-Gorlin) syndrome with taurodontism
}

\author{
This paper is a case report of a \\ 10-year-old girl with focal dermal \\ hypoplasia (FDH) who presented for \\ dental care. She displayed many of \\ the well-documented oral features \\ associated with this syndrome. \\ Additionally, she had taurodontism \\ of a permanent mandibular molar. \\ Although taurodontism affected only \\ one tooth in this patient, the presen- \\ tation of taurodontism with FDH has \\ not been reported previously.
}

$\Delta$ Ithough the earliest case report on Goltz-Gorlin Syndrome or focal dermal hypoplasia (FDH) was published by Jessner in 1921, FDH was first described as a definitive syndrome in $1962 .^{2} \mathrm{FDH}$ is characterized by widespread anomalies of structures derived from both ectodermal and mesodermal tissues. As a result, there are abnormalities of the skin, eyes, central nervous system, musculoskeletal system, and oral structures. $^{2}$

The etiology of FDH is unknown. Approximately 160 cases have been reported worldwide. ${ }^{3}$ Of the reported cases, 95\% have been sporadic, and only $10 \%$ of cases have affected males $($ male:female ratio $=11: 150) .{ }^{3}$ In those cases with a familial history, it has been suggested that the disorder is X-linked-dominant, and usually the transmitting parent is affected with a mild form of the condition. Additionally, FDH is lethal in males and causes significantly reduced fertility in females. A marked underdevelopment of the dermal connective tissues and replacement of these tissues by fat are the first signs of this disease. Skin defects that have been reported include linear hyperpigmentation or hypopigmentation, linear deposits of superficial fat, focal absence of skin, and telangiectasis. Ocular abnormalities such as chorioretinal and iris colobomata, strabismus, nystagmus, blocked tear ducts, and microophthalmia have been described. Also, skeletal abnormalities, particularly of the extremities, resulting in a short asymmetric stature and/or scoliosis, may occur. ${ }^{t h}$

Oral abnormalities, which are a regular finding in FDH, have been well-documented and include the following: widespread papillomas of the oral mucosa that extend to the esophageal region; cleft lip and palate; dental anomalies (such as hypodontia, oligodontia, enamel pitting, and delayed tooth eruption); and malocclusion. ${ }^{6,7}$ In most instances, FDH is diagnosed at birth Additionally, there is little definitive information available on the life-span of affected individuals; however, it is expected that the life span would be very much dependent on complicating cardiac and/or renal factors associated with this syndrome.

This paper presents a case report of a female child who presented at birth with many of the abnormalities detailed above, including an additional diagnosis of taurodontism. Soon after birth, this patient's diagnosis of FDH was confirmed after she was referred to a specialized pediatric hospital in Dublin, Ireland.

\section{Case report}

A 10-year-old girl was referred by the plastic surgery department for dental care. Because she was adopted, no family history could be ascertained. Clinically, she presented with extensive skin abnormalities and linear defects of the epidermis, fatty protrusions affecting her face (Fig. 1), arms, and legs, and areas of hyperpigmen- 
tation. In addition, nail defects were noted (Fig. 2). She had congenital nystagmus and glaucoma, with a very large coloboma of her right eye. In early childhood, the patient had numerous elective ocular surgical procedures, and following her last operation at age 5 , her left eye reduced gradually and became phthisical. Although the palate region was not cleft, the function of the soft palate was abnormal, resulting in velopharyngeal incompetence. At 9 years of age, the patient underwent a pharyngoplasty in an attempt to improve speech. Post-surgically, there was a significant reduction in hypernasality and the ability to create normal sounds with good intra-oral air pressure. Overall, this patient's main problems were skin and ocular in nature and, fortunately, were not life-threatening.

The patient's dental anomalies included hypodontia, microdontia, abnormal tooth morphology, and delayed dental development. There were generalized enamel pits noted in both the primary and secondary dentition (Fig. 3), and the root morphology of the permanent dentition was poor. Widespread papillomas of the oral mucosa were present, involving the palatal mucosa and buccal attached gingiva. Also, additional frenal attachments were noted (Fig. 4). The patient had a Class II division I malocclusion with marked rotation of the right maxillary central incisor (Fig. 4).

Radiological findings confirmed the abnormal crown and root morphology of the dentition and the overall delayed dental development (Fig. 5). The maxillary lateral incisors, right permanent canine, and first and second premolars were absent. In the mandibular arch, the central incisors, right permanent canine, and left second premolar were absent. There was no evidence of developing third permanent molars. The maxillary and mandibular bony morphology (as viewed from the panorex) appeared normal. Finally, taurodontism of both mandibular first permanent molars was evident.

The patient's dental anomalies

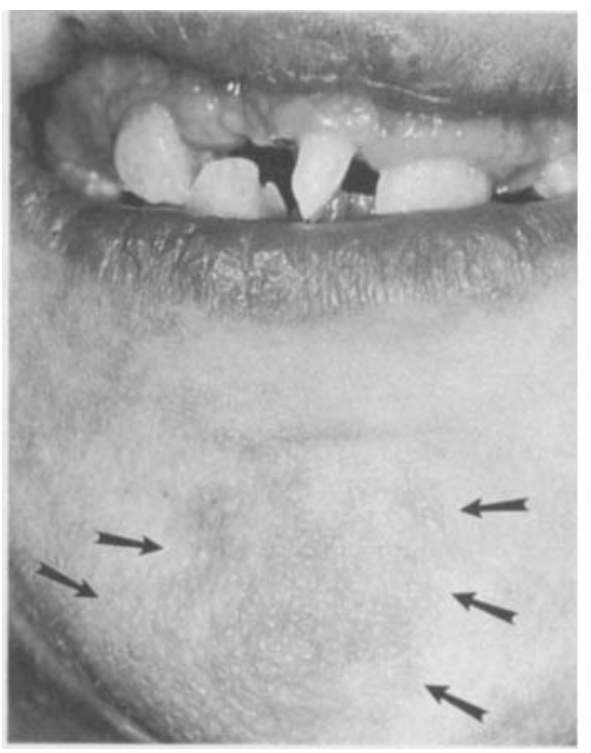

Fig 1. Extraoral lower facial and chin region showing fatty herniation of dermal tissue through linear defects of the facial epidermis.

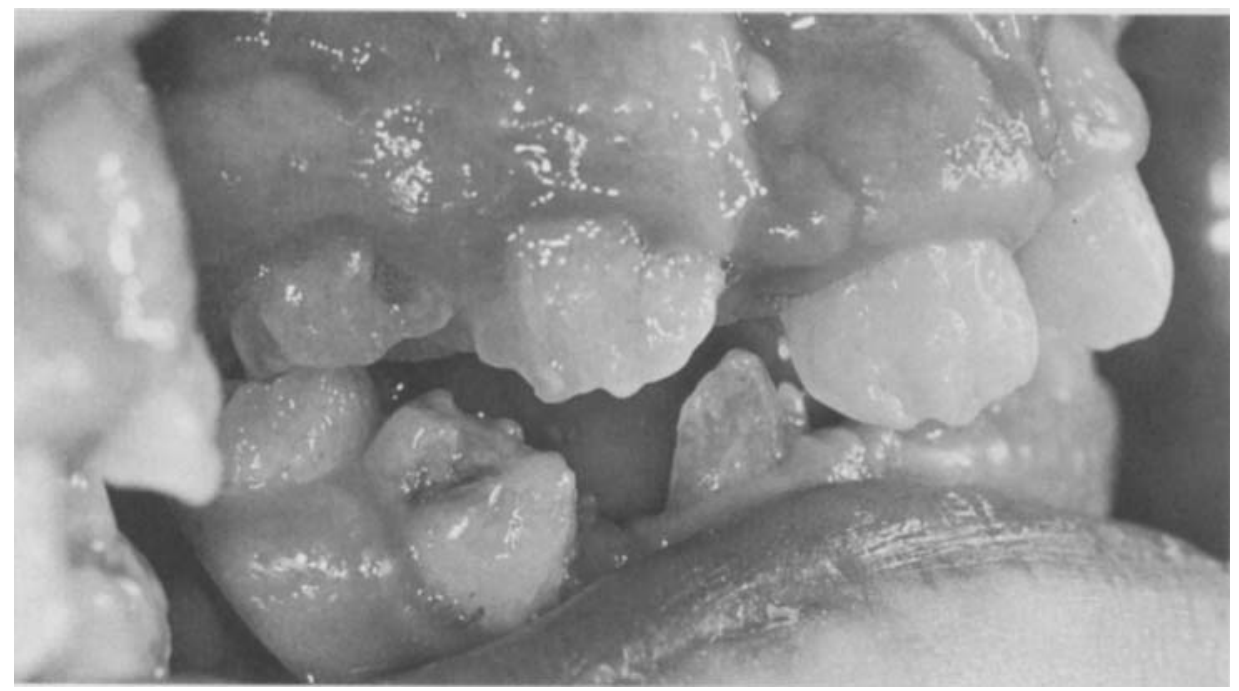

Fig 3. Intraoral view showing microdontia, hypodontia, enamel pitting, abnormal crown morphology, and malocclusion.

were complicated by her poor oral hygiene, gingivitis, and extensive decay of the permanent molars. Overall, the dental management was complicated not only by the esthetic problems arising from the poorly formed, pitted, and maligned anterior teeth, but also by the patient's anxiety and fears toward dental treatment. Following appropriate dental management, however, the gingival and caries problems have been resolved. Stainless steel crowns were placed on all four first permanent molars, and a functional posterior occlusal vertical dimension has been re-established. The maxillary central incisors are currently being rotated by fixed orthodontic appliance therapy. Although the affected molars were restored with stainless steel crowns, the risk of pulpal exposures was increased, and retention of the crowns was made more difficult. Successful restoration of the molar teeth has enabled the patient's functional vertical occlusal dimension to be re-established prior to further restorative 


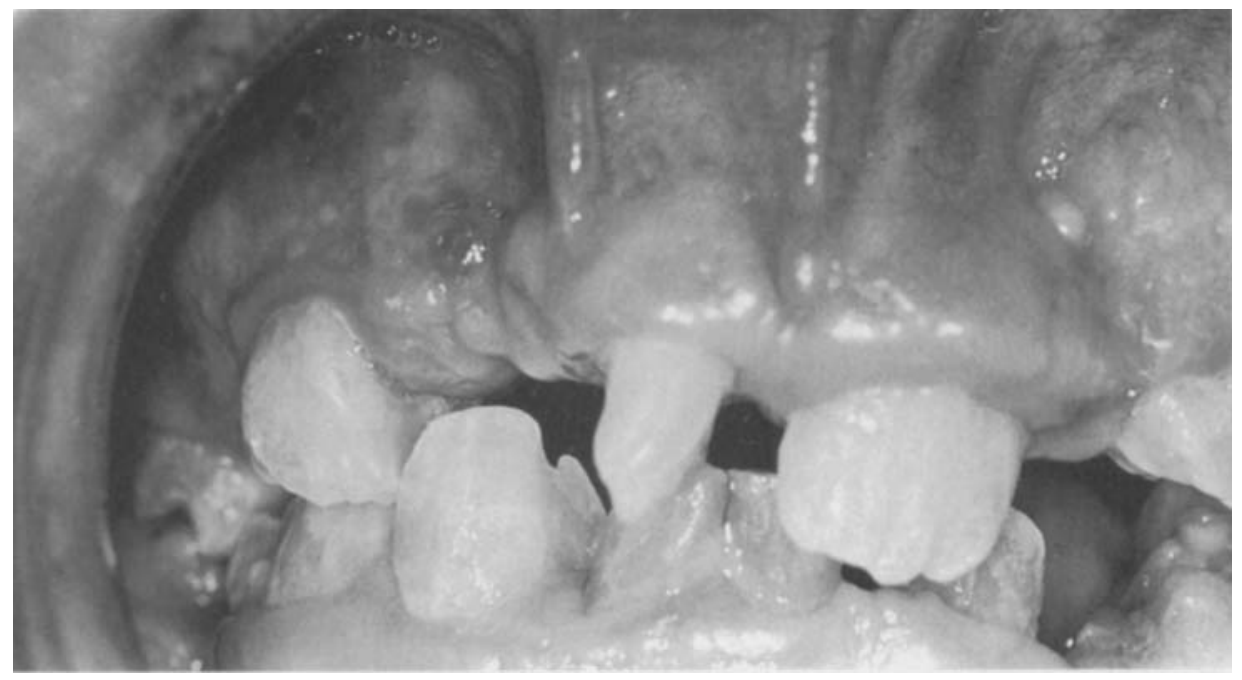

Fig 4. Intraoral view showing additional labial freni and oral papillomas.

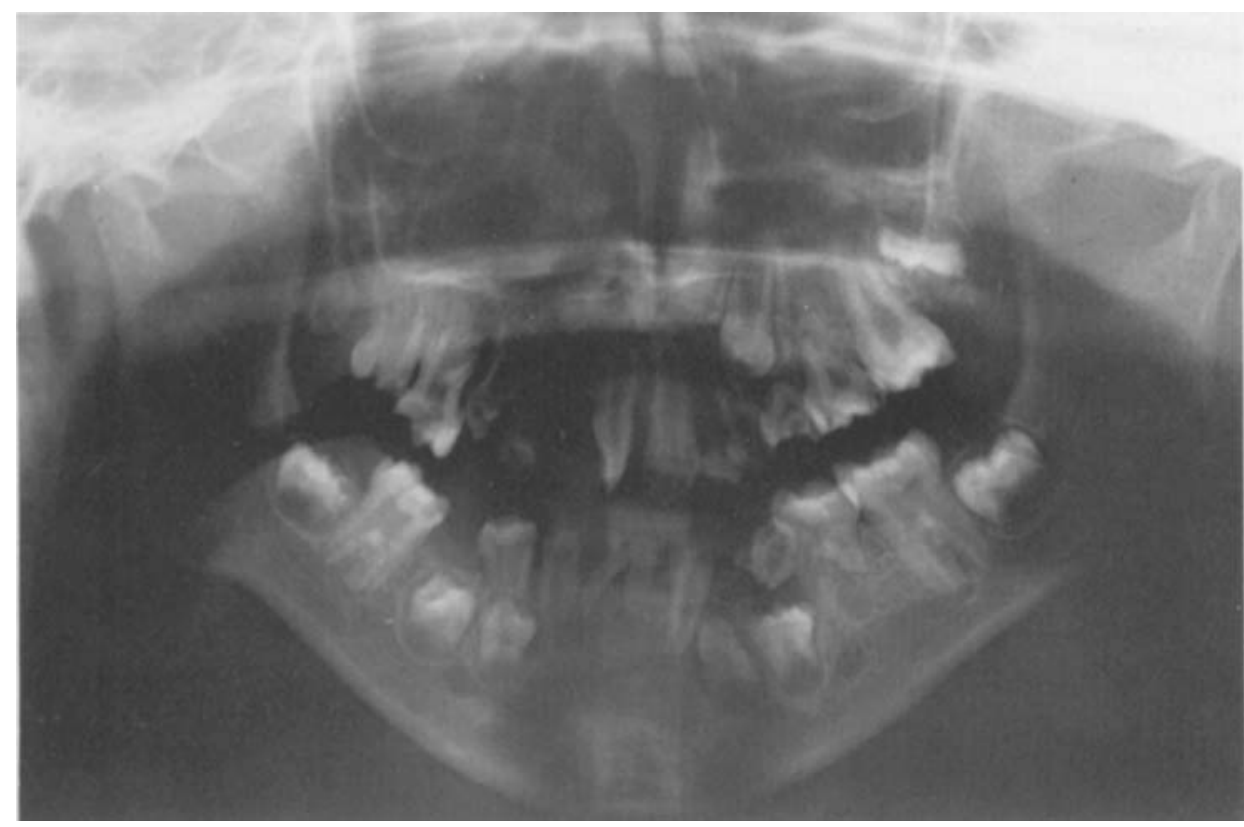

Fig 5. Panorex showing hypodontia, delayed dental development, abnormal crown morphology of both primary and secondary dentition, and taurodontism.

treatment that will follow the fixed orthodontic appliance therapy.

\section{Discussion}

FDH is a rare syndrome with many oral manifestations. The patient in this case demonstrated oral abnormalities such as oral papillomas, abnormalities of the palate, hypodontia, delayed dental eruption, and abnormalities of tooth morphology. Taurodontism is a morphological tooth anomaly characterized by an increased crown-to-root ratio, ${ }^{8}$ and a lack of constriction at the tooth-cervical margin. Permanent molars are usually affected. Radiographs show relatively short roots with large lowlevel pulp chambers. The prevalence of the condition has been reported to be from $2.5 \%$ to $3 \%$ in Caucasians, ${ }^{9}$ although values ranging from $0.57 \%$ to $11.3 \%$ have been recorded. ${ }^{10}$ In addition, there are reports of taurodontism in association with many syndromes, such as Klinefelter's syn- drome, tricho-dento-osseous syndrome, ${ }^{11}$ oro-facial-digital syndrome, and Down's Syndrome. ${ }^{10}$

In the present case report, the taurodontism that was noted on radiographic examination was an additional complication to previously reported oral anomalies associated with FDH syndrome. Long-term dental care will involve further restorations to address the morphological tooth defects and hypodontia. To date, this patient has responded well to simple orthodontic treatment; however, because the root morphology is very poor in these patients, the long-term prognosis for her dentition is guarded, and any additional orthodontic treatment should be avoided if possible.

The authors are in the Orthodontic Department, Western Health Board, Merlin Park Hospital, Galway, Ireland. Dr. Trotman is Assistant Professor, The Department of Orthodontics and Pediatric Dentistry, The University of Michigan, Ann Arbor, MI 48109.

1. Jessner M. Falldemonstration Breslauer dermatologische vereinigung. Arch Dermatol Syph (Berlin) 133:48, 1921.

2. Goltz RW, Peterson WC, Gorlin RJ, Ravits HG. Focal dermal hypoplasia. Arch Dermatol 86:708-17, 1962.

3. Buyse ML. Birth defects encyclopaedia. Vol. 1. London: Blackwell Scientific Publications, Inc., pp 516-7, 1990.

4. Gorlin RJ, Cohen MM, Levin LS. Syndromes of the head and neck. 3rd ed. Oxford: Oxford University Press, pp 472 $5,1990$.

5. Goltz RW, Henderson RR, Hitch JM, Ott JE. Focal dermal hypoplasia syndrome. Arch Dermatol 101:1-11, 1970.

6. Goltz RW. Focal dermal hypoplasia syndrome: An update. Arch Dermatol 128:1108-11, 1992.

7. Mallory SB, Krafchik BR. Goltz syndrome. J Pediatr Dermatol 6:251-3, 1989.

8. Llamas $\mathrm{R}$, Jimenez-Planas A. Taurodontism in premolars. Oral Surg Oral Med Oral Pathol 75:501-5, 1993.

9. Witkop CJ, Keenan KM, Cervenka J, Jaspers MT. Taurodontism: An anomaly of teeth reflecting disruptive developmental homeostasis. Am J Med Genet 4:85-97, 1988.

10. Schalk-Van Der Weide Y, Steen WHA, Bosman F. Taurodontism and length of teeth in patients with oligodontia. J Oral Rehabil 20:401-12, 1993.

11. Seow WK. Trichodentoosseous (TDO) syndrome: Case report. J Pediatr Dent 15:355-61, 1993 . 